\title{
Mujeres en el México postrevolucionario
}

doi: http://dx.doi.org/10.32870/

espiral.v24i68.5250.g5640

\author{
María Candelaria Ochoa Ávalos•
}

El trabajo de cerca de diez años que presenta la doctora Teresa Fernández en este libro denominado Mujeres en el cambio social en el siglo XX mexicano $^{1}$ se integra sin duda en un texto que estamos obligados a leer, porque constata que las mujeres hicimos, hacemos y haremos historia.

Cinco mujeres se nos revelan: Belén de Sárraga, Atala Apodaca, María Arcelia Díaz, Guadalupe Martínez Villanueva y Guadalupe Urzúa Flores. Todas ellas, dice la Dra. Fernández, se nutrieron de tradiciones liberales y en sus trayectorias de vida marcaron a la Revolución y la historia de Jalisco. Ellas (aunque no fueron las únicas), con el trabajo que realizaron, visibilizaron a las mujeres de principios del siglo XX. Ellas ayudaron a la creación de una cultura de la mujer moderna en la época postrevolucionaria mexicana. Al mismo tiempo, los procesos históricos influyeron en sus vida personales, ya que se convirtieron en activistas y feministas, por ello, se puede decir, y la autora lo reitera en el texto, que la política se ve influida por el género, pero también esta influye en la forma en cómo se trata a las mujeres.

Una característica de estas cinco mujeres, y parte de este libro, es que se formaron en tra-

Profesora-

Investigadora del Centro de Estudios de Género, Universidad de Guadalajara, México. mcande.ochoa@gmail. com
I. La Dra. María Teresa Fernández Aceves se hizo acreedora con este trabajo al Premio Clavigero del INAH en 2015.
María Teresa Fernández Aceves (20/4). Mujeres en el cambio social en el siglo XX mexicano. México: Siglo XXI-CIESAS. 
diciones liberales, anticlericales, anarcosindicalistas y de librepensamiento. Con estos principios, confrontaron al sistema liberal que legitimaba la subordinación de las mujeres. Se convirtieron junto con otras en luchadoras por la emancipación de las mujeres, por lograr educación y por ejercer sus derechos sociales y políticos. Fueron defensoras del combate del Estado a la Iglesia católica. Estaban convencidas que debía existir la separación de estos dos como un principio para la laicidad.

La autora parafrasea a Joan Scott cuando dice que hay que visibilizar a las mujeres para ir más allá de su visión victimizada y subordinada o su excepcionalismo, y no pierde de vista el análisis sobre la agencia, es decir, la capacidad de cada una para participar en las movilizaciones y las discusiones sobre el poder, la política y la ciudadanía, y cómo han negociado y transformado los papeles de género no sólo en una sociedad determinada, sino también en su vida cotidiana.

Cada una de estas cinco mujeres marcaron agenda: fueron actoras de la conformación del nuevo Estado mexicano, participaron en la educación pública, fueron entes activos en la formación del movimiento obrero, participaron activamente en la reforma agraria, forjaron el movimiento feminista de la época y fueron parte activa en la conformación de partidos políticos. Y, dice la Dra. Fernández: las cinco mujeres en el cambio social aportan de manera significativa a la historiografía del movimiento de mujeres en México, a la agencia y al empoderamiento femenino y feminista.

Por cierto, a cien años del primer congreso feminista realizado en Mérida el 13 de enero de 1916, habría que plantear un debate sobre los logros y retrocesos de las mujeres en el México contemporáneo. Sobre todo porque a pesar del gran activismo que tuvieron las mujeres y su lucha contra los oscurantismos, el Estado postrevolucionario les tuvo miedo. Por ello, se construyó un modelo de comportamiento burgués 
y apolítico de las mujeres, en donde el modelo masculino caracterizó al "ciudadano" como activo, combativo, franco, forjador, honrado, justo, liberal, noble, racional, patriota, productivo, protector, triunfador, valiente y viril; ese era el producto del nuevo Estado mexicano.

Las mujeres no sólo no obtuvieron el voto en el Constituyente de 1917, sino que muchas veces sus luchas fueron "silenciadas" porque trastocaban el orden social y los modelos de género. Quizá, cien años después, la lucha feminista sigue vigente porque mantiene su intransigencia para visibilizar y politizar el ser "femenino" que trastoca el orden social y los modelos de género.

Aun cuando la Revolución no les hizo justicia y no las emancipó, sus biografías nos permiten conocer a mujeres que con sus propios recursos cuestionaron las relaciones de poder, pero que cada vez estas se tejieron de manera más compleja para reconocerlas como ciudadanas.

La Dra. Teresa Fernández da vida a mujeres caracterizadas como libertarias y librepensadoras. Belén de Sárraga creía en la igualdad entre los sexos en las esferas intelectuales y la política. Provocó con su inteligencia, que se dijera que eso "no era propio de su sexo", y algunos motes a los que se hizo acreedora fueron obrerista, feminista, comunista, campeona de su sexo, ángel caído, ejemplo representativo de las maldades (el socialismo y feminismo), cerebro macho, dama verbosa y mujer viril, entre otros.

Atala Apodaca se destacó por el papel que tuvo en la lucha revolucionaria de Jalisco, apoyando la candidatura de Madero, y siendo por tanto antirreeleccionista. Promovió el ideal de "la mujer moderna", que debía ser liberal, educada e independiente, en una sociedad secular. Trabajó por los postulados de la Revolución mexicana, dando conferencias sobre el movimiento obrero en las plazas públicas, ganándose el título de "conferencista de la Revolución". 
María Arcelia Díaz fue defensora férrea de los derechos de las trabajadoras en la industria textil y fue nombrada "la bolchevique", muchas veces amenazada de muerte por el trabajo que desempeñaba. Aun cuando no se conoció por escrito, sus demandas básicas se referían a lo que hoy denominamos "política social": pago de un salario mínimo y horas extras, condiciones de salud en el trabajo, derecho a la vivienda, entre otras. Creó el Circulo Feminista de Occidente, en el que se formaban mujeres.

Guadalupe Martínez -de quien quizá por un lapsus editorial no incluyen en el índice el capítulo que le correspondefue una mujer que se convirtió en representante intelectual de las trabajadoras nixtamaleras, zapateras, domésticas y costureras; colaboró en la alfabetización y capacitación política de las mujeres y desafortunadamente se alió con el ala conservadora de la Confederación Obrera de Jalisco, encabezada por el cacique Heliodoro Hernández Loza, con quien estableció una relación afectiva que les permitió a ambos, configurar su poder y control sindical, llegando incluso a la expulsión y encarcelamiento de disidentes. Guadalupe Martínez se convirtió en icono de las mujeres priistas de Jalisco y reprodujo el modelo tradicional de familia y mujeres.

Guadalupe Urzúa, por su parte, beneficiaria del voto de las mujeres, fue cuatro veces diputada, logró reconocimiento social por convertirse en una promotora de la reforma agraria y de servicios sociales para su comunidad. Fue considerada como una "mujer fatal", ya que se consideraba que por su belleza conseguía ciertos beneficios. En estas últimas dos biografías se puede observar a mujeres que no amenazaron al sistema, sino que se convirtieron en sus aliadas, primero porque no cuestionaron el poder patriarcal y también porque sus mecanismos de trabajo engranaron con la configuración del sistema priista. 
En cambio, las tres primeras, Belén, Atala y Arcelia, fueron vistas como transgresoras del estereotipo femenino porque rompían con el ideal "femenino" de sumisión, madres, esposas y apolíticas, lo que generaba temor y ansiedad para el sistema y ellas, al desafiar ese pensamiento, se convirtieron en una amenaza.

Las cinco biografías dejan mucho aprendizaje y muchos temas pendientes: Belén de Sárraga y la diferencia sexual como distinción de desigualdad; Atala Apodaca y la enseñanza escolar y cívica para las mujeres, que sigue siendo una demanda; María Arcelia Díaz y los derechos sociales de las trabajadoras en la industria textil; María Guadalupe Martínez Villanueva en el sindicalismo obrero, que hoy es el menor defensor de los trabajadoras; y María Guadalupe Urzúa Flores desde su lucha local por la reforma agraria, no sólo inacabada, sino derrotada. Cinco espacios complejos, netamente políticos, en los que estas cinco mujeres complejizan su participación, apuntan a un primer feminismo que luchaba porque se reconocieran sus derechos, a prácticas complejas del ser mujer, a un cuestionamiento de los procesos revolucionarios y sus logros hacia los menos beneficiados, pero, sobre todo, al fortalecimiento de una política de Estado nacionalista, que hoy cada vez más es considerada como "dañina" a la nación.

Las cinco biografías en este extraordinario libro ayudan a pensar el presente. Permiten visibilizar líneas de debate para el futuro: los logros, retrocesos y, sobre todo, pensar hacia dónde vamos. Hoy permanece la amenaza de la violencia política: aun cuando hoy podemos ser legítimamente diputadas, presidentas municipales, regidoras, maestras, médicas, abogadas, etc., hay todavía quienes no pueden ejercer su cargo, se pone en cuestionamiento la honra de manera permanente, la lucha intragénero y la pelea por el reconocimiento del "otro masculino", la construcción de 
liderazgos y su reconocimiento, la democratización de los sindicatos, entre muchos otros asuntos.

Estas mujeres serán conocidas y reconocidas por su labor, pero no será fácil. Por ejemplo, fue hasta 2013 que se inscribió el nombre de Atala Apodaca en el muro del Ayuntamiento de Guadalajara, esto no para "adornar" un lugar, ni para que sólo ocupara un lugar en una pared casi llena de nombres de hombres y casi vacía de nombres de mujeres: fue para visibilizar a muchas mujeres que forjaron el México postrevolucionario.

Joan Scott dice que las mujeres han sido invisibilizadas porque muchas desafían el stablishment. Lo que hicieron las biografiadas, y lo que hacen muchas, servirá como un ejemplo para construir un futuro más justo e igualitario.

La autora termina diciendo que un reto importante del feminismo ilustrado y teórico es realizar estudios sobre el caciquismo de las mujeres. Sin duda, ese eje está pendiente, pero este libro apunta hacia allá. 